\title{
A Tune for Lowering Lattice Friction
}

\section{Placing an acoustic source on a sample could eliminate the energy dissipation caused by atomic defects moving through the solid.}

\section{By Rachel Berkowitz}

W

hen a crystalline material deforms inelastically, atoms in the crystal lattice lose some of their nearest atomic neighbors and acquire new ones. Those atomic mismatches, or defects, propagate throughout the lattice to release internal energy or to alleviate stress from an external load. As the defects climb up and fall down energy barriers in the lattice's periodic structure, they lose energy by radiating heat. That dissipation, or friction, is beneficial when the material is used to dampen shocks; but the self-heating can cause damage in materials exposed to multiple cycles of loading. Nikolai Gorbushin from ESPCI Paris and colleagues now explain how acoustic waves directed into the material from outside could fully suppress the friction caused by moving crystal defects [1].

At the macroscale, external sources of ultrasound may reduce friction in a solid through acoustic unjamming as, for example, in the remote triggering of earthquakes. Gorbushin and his colleagues applied the same idea to microscale lattice friction.

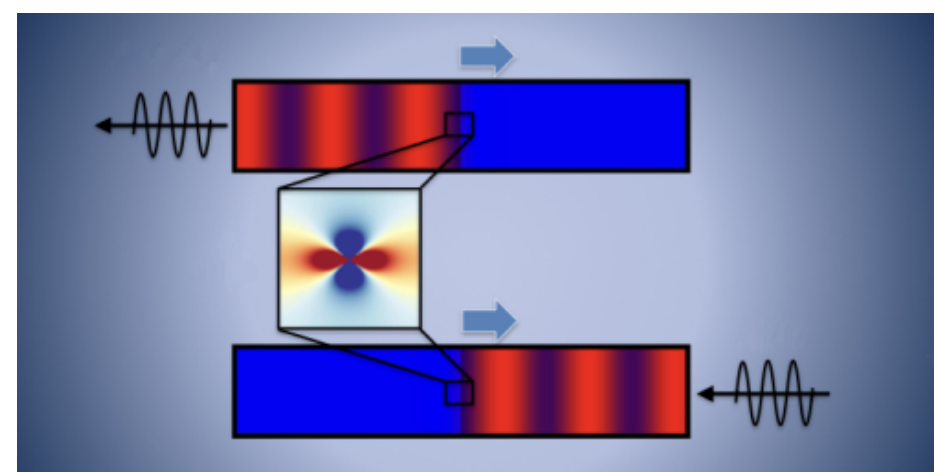

Credit: L. Truskinovsky/ESPCI Paris
The researchers modeled how an ultrasonic source, placed at the edge of a crystalline sample, could steer the defects as they move through the lattice. Their calculations showed that by choosing particular frequencies and amplitudes, the generated sound waves can assist defects in climbing up energy barriers and sliding off gently, without producing any radiation. The models show that the technique can turn a crystal into a system completely free of internal friction.

The finding could help inform designs for suppressing friction associated with different types of crystal defects. It could also help researchers develop metamaterials that are intended to transmit mechanical information by moving topological defects.

Rachel Berkowitz is a Corresponding Editor for Physics based in Seattle, Washington, and Vancouver, Canada.

\section{REFERENCES}

1. N. Gorbushin et al., "Frictionless motion of lattice defects," Phys. Rev. Lett. 125, 195502 (2020). 